\title{
Study on a High-Efficiency Jet Mode of Static Gas Wave Refrigeration
}

\author{
Weihua $\mathrm{Xu}^{1, \mathrm{a}}$, Jiupeng Zou ${ }^{2, \mathrm{~b}}$, Jiao Cheng ${ }^{3, \mathrm{c}}$ and Yuqiang Dai ${ }^{4, \mathrm{~d}}$ \\ 1,2,3,4 School of Chemical Machinery, Dalian University of Technology, \\ Ganjingzi District, 116023, Dalian, Liaoning, P. R. China \\ aweihuaxu361@163.com, bzoujp@dlut.edu.cn, 'chengjiaodut@126.com, ddaiyuqiang@dlut.edu.cn
}

Keywords: Gas wave; Refrigeration efficiency; Multi-stage oscillation; Time-averaged jet flow Abstract. With the external micro-jet to stimulate main jet flow to generate multi-stage oscillation and get time-averaged pulse, a novel high-efficiency jet mode of static gas wave refrigeration is proposed. Investigation made by CFD indicates that the loss of total pressure is less than $45 \%$ of that of the self-excited oscillation, jet flow total pressure retention rate increases from less than $70 \%$ to nearly 90\%. On this basis, the external excited multi-stage oscillation performance is investigated and time-averaged multiple jets are acquired. The new mode overcomes the inherent defect that the time of the exhausting phase is seriously insufficient and the pressure in the gas wave tube is excessive, also makes up for the shortcoming that the jet period and quantity of flow injected into every tube are not the same. Meanwhile, the refrigeration efficiency of gas wave tube could be increased more than $15 \%$ for the reason of the characteristics of time-average.

\section{Introduction}

Static gas wave refrigerator [1] can achieve refrigeration depending on oscillating jet flow periodically injected to every fan-shaped gas wave tube. It can operate with no moving components and not be blocked by the ice and particle. Therefore, the static gas wave refrigerator has characteristic of higher reliability and high pressure bearing ability. However, the inefficiency still limits its commercial application.

Coanda effect [2] and self-excited function is the working foundation of the static gas wave refrigerator. However, the oscillating distribution of the high speed main jet flow leads to a massive energy loss. More energy of main jet flow is expensed for stimulating main jet flow to switch. Numerical simulation shows that the total pressure retention rate of self-excited jet oscillation is only about $60 \%$ to $70 \%$.

When swing jet is injected into two tubes type refrigerator, the time of the injecting phase and the exhausting phase are the same. There would be a significant increase of the pressure of the tubes and a great decrease of effective expansion ratio. So, the temperature drop is small and refrigeration efficiency is low. For multi-tube type refrigerator, although the time ratio of the tubes is less between the injecting phase and the exhausting phase, the quantity of flow injected into these tubes is nonuniform. The refrigeration efficiency of the bilateral tubes has no significant increase. The tubes close to the middle position (named close middle tubes) respectively receive jet flow by the means of executing alternately between long period and short period. Therefore, these also can't reach a high efficiency.

Aiming at the problem that the loss of self-excited oscillation [3] is serious, a novel oscillating type(named the external excited oscillation) that two antiphase micro-jets with the same pressure stimulate main jet flow by turns to realize oscillation, is proposed and investigated. Because of the pressure of the external excited flow exceeds that of self-excited flow and can be accelerated with the aid of pressure gradient to keep up with the main jet flow, the energy of main jet flow not be consumed observably. Besides its high-efficiency, the oscillating type can be started easily and can operate at adjustable frequency.

In order to avoid inconformity of the jet period and quantity of flow injected into every gas wave tube, oscillating jet branches are stimulated again by other micro-jet flow to make every branch jet is 
divided into two, so even jets with equal period and quantity of flow are obtained. Therefore, the novel jet mode named external excited two-stage time-averaged jet mode is proposed and investigated [4].

\section{Structure Model and Numerical Simulation}

The respective energy loss of the external excited and self-excited oscillation is calculated and summarized and the refrigeration efficiency of gas wave tube in different jet modes is also computed. The analytical results will lay the foundation for further experimental research.

Structure of Traditional and Novel Jet. The flow channels of the traditional jet and the two-stage time-averaged jet are shown in Fig. 1. The depth of flow channel much larger than the width of that in order to make external excited flow generate greater force acting on main jet flow, the effect of the upper and lower boundary in the direction of depth on flow is small enough. Compared to the simulation result of three-dimensional model, the numerical calculation of two-dimensional model has enough precision to simulate the unsteady flow of gas channels and save a lot of calculation time.

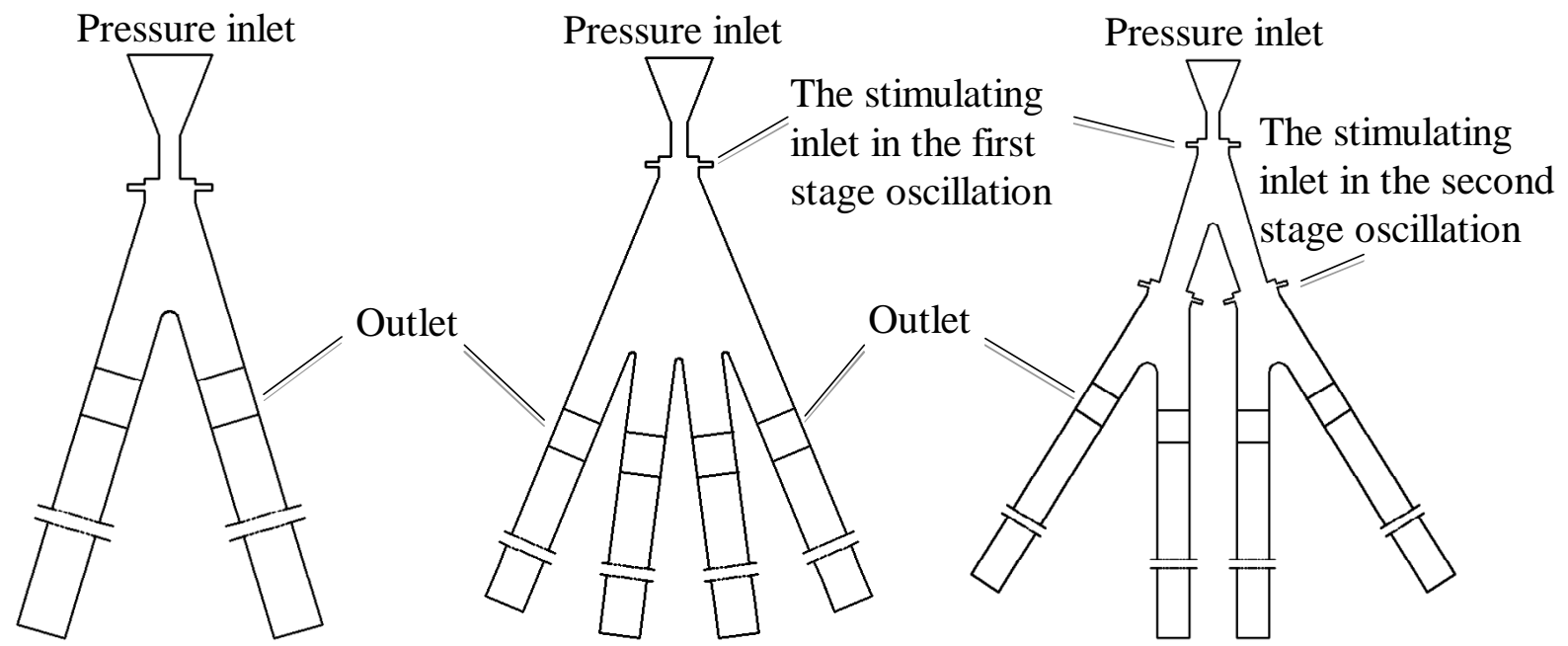

Fig. 1 Structure of the flow channels of the traditional and novel two-stage time-averaged jet

The computational domain is divided into structured mesh by ICEM. The maximum mesh size, based on grid independent test, is $0.2 \mathrm{~mm}$. Moreover along with the direction of velocity, the nozzle adopts denser mesh and the gradually thin mesh is used for gas wave tube. A 2D finite volume code is used to simulate the unsteady flow in gas wave tube. To solve the high velocity turbulence problem of the compressible gas, a realizable $k-\varepsilon$ model [5] is used. Inevitably, there is supersonic flow in the gas wave tube, and the pressure wave will be generated. To capture the waves, Roe's flux difference splitting technique and Monotonic Upwind Scheme for Conservation Laws (MUSCL) [6] discrete scheme as well as Central Difference Scheme for diffusion term are coupled. Implicit integration arithmetic is executed.

The boundary condition can be set as follows: the inlet total pressure of nozzle is $0.36 \mathrm{MPa}$, the outlet pressure is constant static pressure. The slightly trapezoidal pressure boundary is applied to the stimulating inlet (as shown in Fig. 2). In the half period of importing stimulating flow, the peak pressure is equal to the inlet total pressure of nozzle. In the other half period of stopping stimulating flow, the trough pressure is equal to the outlet static pressure. The gas, used in the flow channels and gas wave tubes, is assumed to be ideal gas, and the viscosity $\mu$ is $1.7894 \times 10^{-5} \mathrm{~kg} / \mathrm{m} \bullet \mathrm{s}$. The rest is wall boundary. 


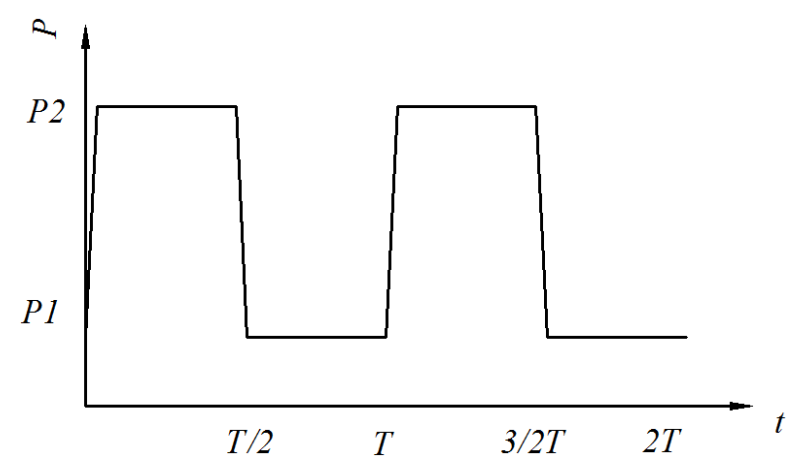

Fig. 2 Pressure curve of the stimulating inlet

The Total Pressure Retention Rate of Different Oscillatory Types. K, the ratio of the total pressure of channel export to total pressure of nozzle, denotes the total pressure retention rate. The higher total pressure retention rate of the oscillation jet means the less the energy loss. The $\mathrm{K}$ of the optimized self-excited oscillation, single-stage and two-stage external excited oscillation in the same parameters are shown in table 1.

Table 1 The total pressure retention rate of the different oscillatory types

\begin{tabular}{cccc}
\hline $\begin{array}{c}\text { Oscillatory } \\
\text { type }\end{array}$ & $\begin{array}{c}\text { Self-excited } \\
\text { oscillation }\end{array}$ & $\begin{array}{c}\text { Single-stage } \\
\text { external excited oscillation }\end{array}$ & $\begin{array}{c}\text { Two-stage } \\
\text { external excited oscillation }\end{array}$ \\
\hline $\mathrm{K}$ & $71 \%$ & $88 \%$ & $80 \%$ \\
\hline
\end{tabular}

Table 1 displays the $\mathrm{K}$ of the two-stage external excited oscillation is $8 \%$ less than that of the single-stage external excited oscillation. So the energy loss in the second stage is $8 \%$, which only accounts for about $40 \%$ of the loss of the whole two-stage external excited oscillation. Although the jet flow finishes oscillation twice, the absolute value of total loss of the two-stage oscillation is over $9 \%$ less than that of self-exciter oscillation.

The Duty Cycle of Gas Wave Tube in Different Jet Modes. The duty cycle is the ratio of the time of injecting gas to circulation period. In order to investigate the effect of the different jet modes on the refrigeration efficiency of gas wave tube. The different jet modes are shown as follows: The time of injecting gas in every tube is $1 / 2$ of the total period (named single-stage time-averaged jet). The time of injecting gas in every tube is $1 / 4$ of the total period (named two-stage time-averaged jet). The time and period of injecting gas in every tube are different (named fan-shaped unequal period jet). The duty cycle of gas wave tube in different jet modes are shown in Table 2.

Table 2 The duty cycle of gas wave tube in different jet modes

\begin{tabular}{|c|c|c|c|c|c|}
\hline \multirow{3}{*}{ Modes } & \multirow{3}{*}{$\begin{array}{c}\text { Single-stage } \\
\text { time-averaged jet } \\
{[\mathrm{s}]}\end{array}$} & \multirow{3}{*}{$\begin{array}{c}\text { Two-stage } \\
\text { time-averaged jet } \\
{[\mathrm{s}]}\end{array}$} & \multicolumn{3}{|c|}{ Fan-shaped unequal period jet [s] } \\
\hline & & & \multirow[t]{2}{*}{ Bilateral tube } & \multicolumn{2}{|c|}{ Close middle tube } \\
\hline & & & & $\overline{\text { Long period }}$ & Short period \\
\hline Duty & $41.80 \%$ & $16.75 \%$ & $41.80 \%$ & $4.125 \%$ & $4.125 \%$ \\
\hline Period & $\mathrm{T}$ & $\mathrm{T}$ & $\mathrm{T}$ & $0.541 \mathrm{~T}$ & $0.459 \mathrm{~T}$ \\
\hline
\end{tabular}

Table 2 indicates the duty cycle of every tube for two-stage jet mode is less than half of that of single-stage jet mode, which means that the exhausting time of the two-stage jet mode is over two times of what the latter is. For fan-shaped unequal period jet mode, the bilateral gas wave tubes own the high duty cycle and equal jet period with the single-stage jet mode. The close middle tubes possess the low duty cycle and the different jet periods, which mean the interval of the first jet flow is short and the interval of the second jet flow is long in an integrated circulation. Because of the swing of jet flow, the jet frequency of the close middle tubes is two times of that of the bilateral tubes. 
The Refrigeration Efficiency of Gas Wave Tube in Different Jet Modes. The same structures, computing grids, gas parameters and diverse jet modes are used to calculate the refrigeration efficiency of gas wave tube. The inlet total pressure of gas wave tube transforms periodically by way of trapezoidal graph with the change of the duty cycle in different jet modes. The peak and trough pressure are determined by the average pressure of channels in injecting phase and exhausting phase. The original temperature is $300 \mathrm{~K}$ and the outlet pressure is $0.18 \mathrm{MPa}$. The length of the gas wave tube is $3000 \mathrm{~mm}$, the inside diameter of the tube is $8 \mathrm{~mm}$. The structure and mesh of the gas wave tube are shown in Fig. 3.

Pressure inlet

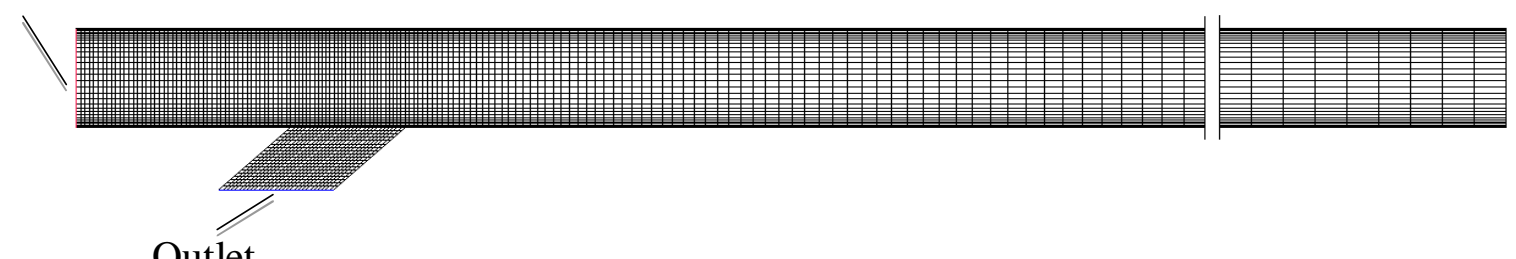

Outlet

Fig. 3 Structure and mesh of the gas wave tube

The isentropic refrigeration efficiency is the main performance index to evaluate the refrigeration characteristic of the gas wave tube. When the working medium is regarded as ideal gas, the ratio of the actual enthalpy drop to the theoretical enthalpy drop is defined as the refrigeration efficiency of the gas wave tube [7]. It is shown in Eq. 1.

(1)

$$
\eta=\frac{\square H_{r}}{\square H_{i}}=\frac{T_{i}-T_{o}}{T_{i}\left[1-\left(\frac{1}{\varepsilon}\right)^{\frac{\gamma-1}{\gamma}}\right]} .
$$

In this formula, $T_{i}$ denotes the temperature of gas in the high pressure inlet. $T_{o}$ denotes the temperature of the exhausting cooling gas. $\varepsilon$ is expansion ratio. The refrigeration efficiency is calculated through monitoring the change of the temperature of outlet.

In order to compare the refrigeration performance of gas wave tube in different jet modes, curves between the isentropic refrigeration efficiency and the jet flow frequency are shown in Fig. 4. The refrigeration efficiency is calculated by the way of mass-weighted average.

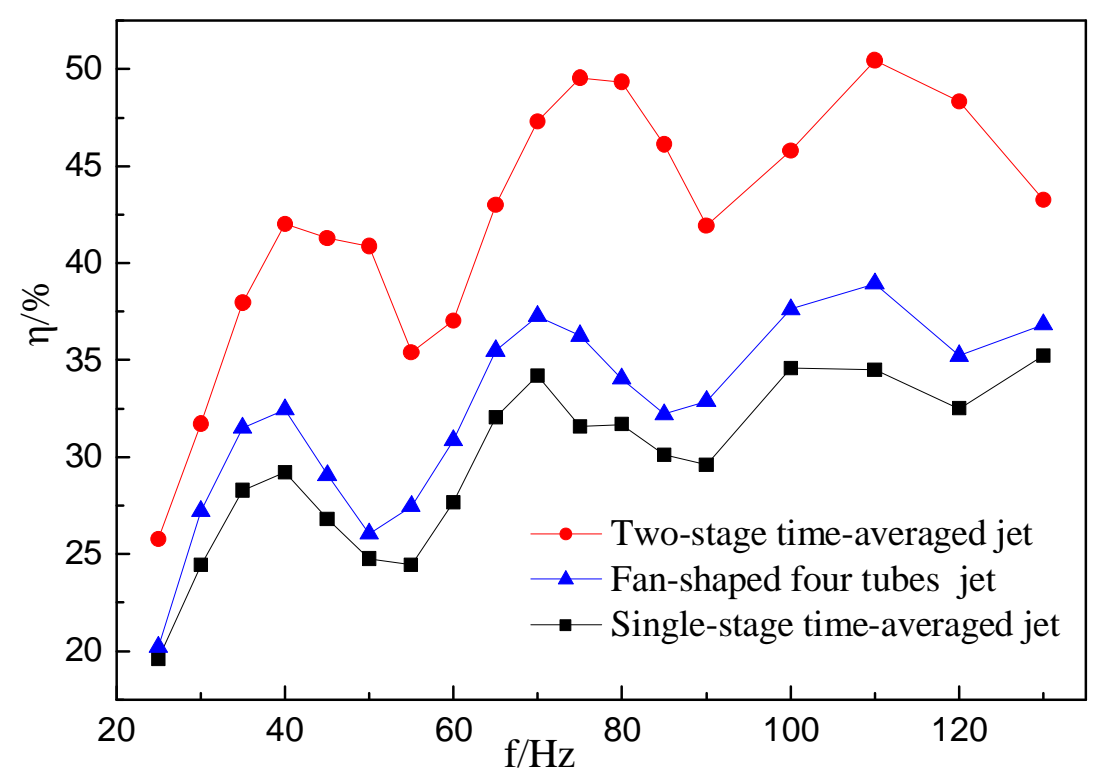

Fig. 4 The curves of refrigeration efficiency in different jet modes 
Figure 4 indicates the refrigeration efficiency of gas wave tube in fan-shaped four tubes jet mode increase by $2 \% \sim 3 \%$ than that of the single-stage time-averaged jet mode. The range is not remarkable. But, the efficiency of the tube in the two-stage time-averaged jet mode increases by approximate $20 \%$.

The refrigeration efficiency varies with the jet flow frequency. It is determined by the matching degree between pressure wave moving and the length of gas wave tube [8].

When the number of fan-shaped tubes increases to five tubes, the swing speed of jet flow is maximal at the position of the middle tube, the quantity of flow and duty cycle of jet flow is very small. So, the tube does not contribute to refrigeration efficiency much. For the close middle tubes, the more far away from the middle position, the more serious the inconsistency of the jet period gets. The refrigeration efficiency of gas wave tube is near between the fan-shaped five tubes and four tubes jet mode. So increasing the number of the tube has a minimal effect on the refrigeration efficiency. Furthermore, the more the quantities of the tube are, the bigger the swing angle become. Because the swing range is relatively reduced, the jet flow may not inject into the bilateral gas wave tubes.

\section{Conclusions}

The loss of total pressure of the external excited jet flow oscillation is remarkably less than that of the self-excited oscillation. And the loss of the second stage oscillation is less than half of the loss of the first stage oscillation. The external excited oscillation can keep more jet energy. Hence it is very much suitable that jet is distributed into the wave tubes by the external excited oscillation instead of self-excited oscillation of the distributary of main jet flow.

The jet mode has an obvious influence on refrigeration efficiency of every wave tube of static gas wave refrigerator. The refrigeration efficiency of the two-stage time-averaged jet mode is markedly higher than that of the single-stage time-averaged and fan-shaped four or five tubes mode. The mean difference exceeds $15 \%$.

Compared to the other jet modes, the novel two-stage time-averaged oscillating jet with external excited oscillation should be the first choice for the static gas wave refrigerator.

\section{Acknowledgements}

This work was financially supported by the National Natural Science Foundation of China (51276026).

\section{References}

[1] W. Lu, D.P. Hu. Refrigeration. No.4 (2002), p. 19

[2] C. E. Seaton, H. Henein, M. Glatz. Pouder Metallurgy. Vol.30 No.1 (1987), p. 33

[3] U Gebhard, H Hein, Schmidt. J Micromech Microeng. Vol.6 (1996), p. 115

[4] J.P. Zou, Y.Q. Dai, X.W. Liu, D.P. Hu, C. Zhu, P.Q. Liu. CHN. Patent 201410484604.8 (2014)

[5] J.A. Fernández, J.C.Elicer-Cortés, A.Valencia, M.Pavageau, S.Gupta. International Communications in Heat and Mass Transfer. Vol.34 No.5 (2007), p. 570

[6] D.Y. Li: The fluid dynamics methods in two-dimensional unsteady flow (science press, CHN 1987) (In Chinese)

[7] H.R. Yu. Journal of the School of Engineering of Dalian. Vol.23 No.4 (1984), p. 1 (In Chinese)

[8] X.Q. Zhu. Journal of the institute of light industry of WuXi. No.2 (1993), p. 135 (In Chinese) 